\title{
Evaluación de riesgos en investigaciones en Psicología y disciplinas afines
}

\author{
Risk assessment in research in Psychology (and related disciplines) \\ Claudia Estrada \\ "Centro de Estudios en Psicología Social (CEPS), \\ Facultad de Educación y Ciencias Sociales, Universidad de Magallanes \\ claudia.estrada@umag.cl
}

\begin{abstract}
RESUMEN
El ensayo intenta presentar algunas ideas generales que permitan el abordaje de la evaluación de riesgos en las investigaciones en Psicología y otras disciplinas que realizan investigación a través, en o con seres humanos. Actualmente la legislación indica que las investigaciones de este tipo deben ser sometidos a evaluación ética por Comités de Ética Científico (CEC), que se rigen por la normativa generada para la investigación biomédica. Esta situación implica que profesionales de las ciencias médicas, se involucran habitualmente en la revisión ética de proyectos en disciplinas como la psicología, intentando asimilar las características de lo biomédico y lo psicosocial. Se discute en torno a las dificultades que esto representa y se presentan una serie de ideas en la dirección de especificar los aspectos que diferencian a las ciencias humanas y sociales en lo que respecta a la determinación del potencial daño producto de la participación en estudios de esta área y sus consecuencias a mediano y largo plazo. Sin pretender ser exhaustivo, el presente trabajo intenta ser un punto de partida para la reflexión en torno al riesgo de las investigaciones en psicología y disciplinas afines.
\end{abstract}

Palabras clave: ética investigación, Ciencias humanas y sociales, riesgo y daño psicosocial.

\begin{abstract}
The essay tries to present some general ideas that allow the approach of the risk assessment in the investigations in Psychology and other similar disciplines that realize


research through, in or with human participants. Legislation currently indicates that such research should be subject to ethical consideration by Scientific Ethics Committees (CEC), which are governed by the regulations generated for biomedical research. This situation implies that professionals of the medical sciences are usually involved in the ethical review of projects in disciplines such as psychology, trying to assimilate the characteristics of biomedical and psychosocial. It discusses the difficulties that this represents and presents a series of ideas in the direction of specifying the aspects that differentiate the human and social sciences on the determination of the potential damage caused by the participation in studies of this area and its consequences in the medium and long term. Without pretending to be exhaustive, the present work tries to be a point of departure for the reflection related to the risk of the investigations in psychology and related disciplines.

Key words: Ethics research, human and social sciences, risk and psychosocial damage.

\section{CONCEPTOS CENTRALES}

Como lo señala el código de ética de la APA (American Psychological Association, 2010), los psicólogos deben comprometer esfuerzos para incrementar el conocimiento científico que conduzcan a aumentar la comprensión de las personas como tales y de su comportamiento. Dicha búsqueda tiene un objetivo fundamental, que es la de mejorar el bienestar de los individuos, organizaciones y la sociedad. Esta acción debe realizarse en el respeto y protección de los derechos humanos y civiles, y en la consideración de la importancia de la libertad de expresión y de indagación.

Un importante desafío de la ética de la investigación con seres humanos en nuestro país, es el desarrollo de cánones específicos para las disciplinas que la realizan entre las cuales se encuentra la psicología. Actualmente, la legislación nos orienta, en consideración de la ausencia de normativa particular, al uso de las normas y leyes propuestas para las ciencias de la salud y, en general, para la investigación biomédica. La investigación en dichas áreas suele comportar exámenes físicos, el uso de procedimientos que pueden ser dolorosos, el ensayo de nuevos medicamentos y artefactos. Cuando se trata de la investigación en Psicología, muchos de estos procedimientos no son parte del método requerido para responder a las preguntas de investigación. Comparativamente, esto ha conducido a los investigadores considerar como "potencialmente menos dañinas" las acciones de entrevistar, aplicar instrumentos de lápiz y papel o practicar la observación del comportamiento. Es este aspecto particular de la disciplina lo que se constituye en el principal desafío de la ética de la investigación científica: evaluar los potenciales riesgos que representan las investigaciones en psicología en sus diferentes áreas de desarrollo.

Esto representa un reto ya que la psicología es una disciplina diversa (neuropsicología, psicología social o clínica, de la salud, etc.) y la definición del riesgo potencial depende de 
una serie de factores no siempre fácil de determinar. Para comenzar, uno de esos factores es la definición del "tipo de estudio" que representa una investigación depende de su objetivo directo que podría clasificarse en tres diseños básicos (Rueda, 2004): a) Con seres humanos, es decir en colaboración con quienes participan en el estudio y cuya presencia es una relación en la que existe un esfuerzo, por parte de la investigación, de retribuirles. Esta retribución sería una consecuencia directa de su participación como, por ejemplo, la entrega de un informe confidencial de sus resultados, el que es recibido como una compensación merecida. También en esta categorización podríamos incluir aquellas investigaciones en las que los objetivos son la respuesta a una demanda de un grupo o comunidad y el conocimiento, en términos de su valor científico, es secundario al de la utilidad y pertinencia que tendrá para aquellos que son parte. b) En seres humanos en la que los participantes y sus intereses son secundarios ya que el centro es la generación de conocimiento, que, aunque pueda o no contribuir al bienestar futuro de las personas, los utiliza como un medio en el presente. c) A través de seres humanos, en que se centra el objetivo en las dinámicas sociales, efectos socioeconómicos e intereses grupales comunitarios. Los participantes son activos ya que actúan como informantes y son, como consecuencia, parte de los equipos de investigación. En todos estos tipos de investigación es posible resguardar los principios de beneficencia, no maleficencia, autonomía y justicia.

\section{El riesgo}

No es posible continuar sin definir el concepto de riesgo y posicionarlo en la Ciencia Psicológica. El riesgo puede ser definido en este contexto, como la posibilidad de daño que ocurre como resultado de la participación en una investigación psicológica. Podríamos considerar que existen diferentes niveles que pueden presentarse simultáneamente en diferentes etapas de un estudio, aunque resulta muy difícil evaluar su impacto a mediano o largo plazo (Rovaletti, 2006). En primer lugar, tenemos el "riesgo nulo", que hace referencia a la inexistencia de riesgo alguno en la participación en la investigación. Los estudios con este nivel de riesgo son escasos y refieren a cuestiones básicas como la discriminación de colores, por ejemplo, o de tiempos de reacción frente a algún estímulo. En segundo lugar, se encuentra el "riesgo mínimo", entendido como aquel que, existiendo, no es mayor al que el participante enfrentaría en su vida cotidiana. Por ejemplo, algunos estudios de psicología social podrían exponer que el uso de la "mentira” podría ser parte de este nivel puesto que algunas ideas (por ejemplo, la creencia en la existencia de lo sobrenatural), se encuentran "rondando" a las personas en su vida habitual. Finalmente encontramos el riesgo "mayor al mínimo", que refiere al riesgo que es mayor al que potencialmente es expuesto una persona cotidianamente y que puede o no tener efecto permanentes.

Los riesgos también puedes ser clasificados según su tipo. Dentro de los potenciales 
tipos asociados a la investigación psicológica encontramos el daño físico, por ejemplo, el que podría producirse como consecuencia de temáticas de estudio tales como delitos, crímenes o violencia doméstica (Weisser-Lohmann, 2012), el daño económico (por ejemplo, temáticas que podrían impacto negativo potencial sobre empleo, estudios u otros) o psicosocial. Este último tipo puede ser considerado como característico de los estudios psicológicos y comprenden una serie de potenciales consecuencias adversas de la participación en un estudio:

A. Revelación de actitudes personales o grupales que pueden conducir a estigmatización, discriminación o prejuicio: Lo que puede parecer una "simple e inocente encuesta" puede convertirse para algunas personas en un hecho que puede determinar el que una persona sea objeto de estigmatización, es decir, que la manifestación de su actitud le conduzca a ser categorizado como miembro de un grupo poseedor de una característica valorada como negativa, aumentando su posibilidad de sufrir discriminación o prejuicio. Algunos estudios que pueden tener estas consecuencias son aquellos que se refieren al comportamiento sexual $\mathrm{u}$ otro tipo de actitud que pertenece a la privacidad. Aunque el respecto a la confidencialidad de la información puede estar asegurada desde el procedimiento de toma y mantención de datos, asistir a un laboratorio en el que se está realizando una investigación determinada puede tener como consecuencia la asignación de una determinada categoría social. En este tipo de daño también encontramos a aquellos estudios que pueden buscar la revelación de actitudes grupales. Por ejemplo, un estudio sobe la personalidad adolescente que tiene como resultado principal que el grupo medido presenta una tasa alta de predisposición delincuente. Si los participantes provienen de un cohorte reconocible (estudiantes de una universidad), la divulgación de dichos resultados puede tener este tipo de consecuencias indeseadas afectando a cada miembro de dicha comunidad.

B. La invasión a la privacidad es considerada, en nuestra legislación, como un delito. La jurisprudencia señala que todo individuo tiene derecho a la intimidad. Esto significa que existe un espacio privado en el que cada persona puede ejercer la opción de ser diferente, ejercer estilos de vida o actitudes poco comunes, y que todo ello es propiedad de las mismas en la reserva que excluye a otras personas (Diaz, 2007). Este espacio sólo se encuentra limitado por la protección de intereses comunes primordiales y la búsqueda de evitar el daño. En este sentido, la investigación de ciertas temáticas podría considerarse un interés común primordial porque permitiría el desarrollo de políticas públicas, pero no es posible hacerlas sin este potencial daño. Por ejemplo, podemos tener la autorización para entrevistar a personas sobre sus historias de vidas asociadas a la emigración. El proceso de recolección de la información puede difícilmente asegurar que la persona no nos entregará antecedentes que no había pensado revelar en un primer momento. En la relación de falsa intimidad que ofrece una entrevista 
psicológica, podría llegar a contarnos detalles de su trayectoria que no necesariamente tienen relación directa con el objeto de estudio pero que, finalmente, podrán hacerle sentir expuesto o revelado a un nivel incómodo. ¿Puede un investigador controlar esta incomodidad? Es poco probable. Su tarea es, muchas veces, permitir al otro expresarse "libremente". Podríamos argumentar que la persona consintió a esta situación y que su información se encuentra protegida. A lo primero podríamos contrargumentar que no hay consentimiento real si no hay "equilibrio de fuerzas", el que no existe cuando un profesional entrenado en indagar en la intimidad se encuentra con otro desprovisto de ese poder. A lo segundo podríamos decir que dicha información está protegida de la divulgación masiva, pero si ha sido puesta en la persona del investigador, quien ahora la conoce y que, quizás, nunca debió conocerla. Por otra parte, puede ocurrir una "ruptura" de la confidencialidad cuando los datos del estudio son recolectados en situación de grupo (ejemplo, grupos focales). Cada uno de los participantes se convierte en un garante de la confidencialidad de lo expresado y compartido de la misma manera que cada uno es un foco potencial de "fuga" de información. En este caso, es necesario poner en conocimiento a los participantes de esta potencial invasión "secundaria” a su privacidad (Arwood y Panicker, 2016)

C. Esto nos conduce a otros potenciales daños del ámbito de lo emocional: los sentimientos de culpabilidad, confusión y vergüenza. ¿Puede una investigación provocar como efecto indeseado algunos de estos sentimientos? La respuesta es sí. Puede hacerlo muy temprano en el proceso cuando la persona es enfrentada a preguntas que considera "delicadas", como por ejemplo la medición de sus hábitos de consumo de alcohol, o más tardíamente, cuando en el proceso de "defriefing” se ve enfrentado a que lo que creyó verdadero y que afirmó (al menos temporalmente) alguna actitud suya, era en realidad una "mentira", parte de una manipulación experimental, o cuando en un proceso de observación de su relación con un niño pequeño, se expone a los que considera el "juicio" de otros, de lo que es su actuar parental. Por esto es que el desarrollo de estos sentimientos negativos, que se vinculan con el sufrimiento, pueden ser consecuencia ya sea directa del mecanismo utilizado como método de responder a las preguntas de una investigación, como consecuencia indirecta de la experiencia de ser "un conejillo de indias".

D. La depresión y la pérdida de confianza: Investigaciones han mostrado que tan solo la manera de presentar las preguntas puede inducir el surgimiento del sentimiento de victimización. ¿cómo podemos negar, entonces, que el estrés y la depresión pueden ser una consecuencia de la participación en un estudio psicológico? Revivir experiencias dolorosas del pasado, actualizarlas en el presente, volver a escucharlas y resignificarlas, puede ser un proceso fuertemente curativo, pero no se da en unas sesiones de entrevistas por bienintencionadas que estas sean. Ese contacto, en dos o tres sesiones, que hablará del trauma colectivo, de 
lo vivido, puede conducir a una re-traumatización, no en el sentido de crear un nuevo trauma, sino que soplar sobre las brasas de un trauma dormido, que vuelve para incomodar, y que puede conducir a la distimia, la depresión o el malestar emocional severo. Incluso en el contexto de un estudio sobre la violencia en la pareja, contestar un cuestionario puede permitir "descubrir" que se es objeto de dicha violencia. Podría discutirse que es un contexto "preventivo”, que permitirá que una persona clarifique la calidad de sus relaciones y tome decisiones "buenas" para sí misma, pero la investigación no busca este efecto y no siempre está preparado para contener los efectos de esta nueva "mirada de la realidad". Es este un riesgo al que cualquiera está expuesto en su vida cotidiana, este el de verse a sí mismo de una nueva manera. ¿Es por esto el que debe permitirse que un participante se vea expuesto a una experiencia que no pidió y que puede vivir como un evento doloroso, de pérdida de confianza, en sí mismo o en los otros?.

E. Estrés y Aburrimiento: Ambos, fuertemente vinculadas ya que el segundo ha sido descrito como una causa potencial de estrés, pueden producirse en el contexto de la participación en estudios en Psicología. Aunque es poco probable que se produzcan efectos permanentes, si pueden ocasionar tensión y desagrado. Algunos investigadores, enfrentados a sus necesidades de productividad, pueden no dudar en aplicar extensos cuestionarios que miden tanto las variables centrales del estudio como muchas otras que, "potencialmente”, les permitirán nuevos análisis y publicaciones de resultados en el futuro. El equilibrio entre la búsqueda del conocimiento y someter a una persona al aburrimiento y el estrés, con su consecuente menor pero existente riesgo de salud, debe lograr un balance que respete el principio de beneficencia y el de no maleficencia.

Cómo evaluar el riesgo:

A. Probabilidad de ocurrencia: Según Santi (2015), el riesgo puede ser evaluado considerando la probabilidad concreta de ocurrencia de un evento potencialmente dañino. Se considerará más grave aquel que tiene una mayor probabilidad (Ej., sentimiento de incomodidad o tensión en una entrevista abarcando información sensible) y menos a aquel que, aunque posible, su ocurrencia podría considerarse una situación excepcional (Ej., trastorno del sueño luego de participar en un experimento en que se debió leer un texto sobre violencia en los estadios).

B. Dimensión del daño potencial: Igualmente, un riesgo puede diferenciarse de otro según la dimensión del daño que puede provocar en el evento de ocurrir. Frente a la misma probabilidad de ocurrencia, un daño podría ser considerado como mayor cuando provocara un efecto adverso masivo (Ej: La ocurrencia de un potencial ataque epiléptico al ser expuesto a estímulos visuales en una investigación neuropsicológica, o la "reactivación" de un síndrome post-traumático en una investigación sobre violencia intrafamiliar). Rid, 
Emanuel y Wendler (2010) proponen siete factores que permiten evaluar la magnitud del daño potencial: 1. El dolor asociado, 2. El esfuerzo que es necesario para mitigarlo, 3. Sus efectos en actividades cotidianas, 4 . La duración del mismo, 5 . El esfuerzo requerido para compensarlo (adaptarse a él) y 6. La carga impuesta para lograr esta adaptación.

C. Permanencia del efecto: Entendida como un elemento que, siendo parte de la dimensión o magnitud del daño, amerita una consideración especial. La evaluación del efecto "residual" de un efecto adverso es uno de los criterios que deben ser considerados como elementos centrales de la evaluación del riesgo (Santi, 2015). En el entendido de que la vulnerabilidad o fortaleza psicológica comporta elementos individuales, es posible prever la extensión temporal de un determinado daño y, así mismo, de la efectividad de las medidas remediales de las que se dispone para enfrentarlo.

Se han realizado esfuerzos por proponer un marco comparativo entre los daños en la investigación biomédica y las Ciencias Sociales, con la intención de favorecer la evaluación de los riesgos como la propuesta por Sieber y Tolich (2003, en Santi, 2015), que considera alguno de los elementos ya mencionados (ver Tabla 1):

Tabla 1: Propuesta de Sieber y Tolich de equiparabilidad de daños (en Santi, 2015).

\begin{tabular}{|c|c|c|c|c|c|}
\hline Magnitud & $\begin{array}{c}\text { Daño en } \\
\text { investigación } \\
\text { social }\end{array}$ & $\begin{array}{c}\text { Comparación con } \\
\text { investigación } \\
\text { médica }\end{array}$ & Duración & Efecto & Tratamiento \\
\hline (1) Insignificante & Humillación & Nausea & Horas & Incomodidad & $\begin{array}{c}\text { Sesión } \\
\text { informativa } \\
\text { posexperimental }\end{array}$ \\
\hline (2) Pequeño & Estrés & Jaqueca & Horas a dias & Perturbación & Relajación \\
\hline (3) Moderado & $\begin{array}{l}\text { Pérdida de una } \\
\text { gran oportunidad }\end{array}$ & $\begin{array}{l}\text { Fractura, } \\
\text { Insomnio }\end{array}$ & $\begin{array}{c}\text { Semanas a } \\
\text { meses }\end{array}$ & $\begin{array}{c}\text { Dificultad para } \\
\text { lograr un } \\
\text { objetivo }\end{array}$ & Rehabilitación \\
\hline (4) Significativo & Estigmatización & $\begin{array}{l}\text { Rotura de un } \\
\text { ligamento }\end{array}$ & Meses & $\begin{array}{c}\text { Desconexión } \\
\text { con la sociedad }\end{array}$ & Terapia \\
\hline (5) Mayor & Brote psicótico & $\begin{array}{l}\text { Pérdida de un } \\
\text { dedo }\end{array}$ & $\begin{array}{c}\text { Semanas a } \\
\text { meses }\end{array}$ & $\begin{array}{c}\text { Terror, cambio } \\
\text { de } \\
\text { personalidad }\end{array}$ & $\begin{array}{l}\text { Medicación, } \\
\text { terapia }\end{array}$ \\
\hline (6) Severo & Depresión & Paraplejia & Décadas & Desesperanza & $\begin{array}{c}\text { Atención médica, } \\
\text { terapia }\end{array}$ \\
\hline (7) Catastrófico & Suicidio & Muerte & Permanente & Fin de la vida & Ninguno \\
\hline
\end{tabular}

Aunque se trata de una propuesta útil y meritoria en su intento de simplificar la evaluación del riesgo, no permite una clasificación clara y definitiva de los daños potenciales descritos anteriormente para las Ciencias Psicológicas ni toma en consideración los factores individuales que en psicología resultan altamente pertinentes. 


\section{CONSIDERACIONES FINALES}

La investigación en Ciencias Psicológicas, tal como en otras disciplinas de las Ciencias Sociales y del Comportamiento no puede clasificarse en términos amplios como investigación "inocua". Los tipos de riesgo que comportan dependen de una serie de factores, algunos mencionados en este breve ensayo. Su importancia o gravedad puede ser similar al observado en la biomedicina o en la investigación en salud con seres humanos, pero su determinación y clasificación, en numerosas oportunidades, exige una particular atención a elementos idiosincráticos difíciles de evaluar en un protocolo de investigación. Esto representa un especial desafío para los miembros de los Comités de Ética Científicos (CEC), quienes no siempre están preparados para evaluar las singularidades de este tipo de investigaciones y cuyo desconocimiento puede llevarlos tanto a sobreestimar como a subestimar el riesgo de daño. Si duda que puede resultar difícil equiparar la muerte física con el sufrimiento psicológico y no hay clasificación alguna que nos conduzca a tener criterios tan definidos que no se requiera el uso del análisis “comprensivo e interpretativo" de los principios generales que guían a la ética de la investigación con seres humanos. En este sentido, este desafío compromete no sólo a los investigadores del área sino a los miembros de los CEC quienes representan a la sociedad en general y a los participantes en particular. La ética de la investigación ya no puede entenderse como una responsabilidad depositada únicamente en los científicos y su compromiso moral (Achío 2003), sino que compartida con quienes asumen el rol de ponderar, con las herramientas que el conocimiento interdisciplinar les proporciona, el potencial riesgo al que es sometida una persona y la justificación que hace este acto algo legítimo.

\section{REFERENCIAS}

Achío, M. (2003). Los Comités de Ética y la Investigación en Ciencias Sociales. Ciencias Sociales, 99, pp 85-95

Diaz, R. (2007). Delitos que Vulneran la Intimidad de las Personas: Análisis crítico del artículo 161-A del Código Penal Chileno. Revista Ius et Praxis, 13(1), 291-314.

Rid, A., Emanuel, E., \& Wendler, D. (2010). Evaluating the Risks of Clinical Research., JAMA, 304(13), 1472- 1479.

Rueda L. (2004). Bioética, fundamentos y dimensión práctica. En Ética de las investigaciones que involucran a seres humanos. Santiago: Mediterráneo.

Sieber, J., \& Tolich, M. (2013). Planning Ethically Responsible Research. Thousand Oaks, California: Sage.

Rovalleti, L. (2006). La evaluación ética en las Ciencias Humanas y/o Sociales. La investigación científica: Entre la libertad y la responsabilidad. Acta bioethica, 12(2), 243-250.

Santi, M. F. (2015). El debate sobre los daños en investigación en Ciencias Sociales. Revista de 
EVALUACIÓN DE RIESGOS EN INVESTIGACIONES EN PSICOLOGÍA Y DISCIPLINAS AFINES.

Bioética y Derecho, 34, 11-25

Weisser-Lohmann, E. (2012). Ethical aspects of vulnerability in research. Poiesis and Praxis, 9, 157-162. 